was immediately diluted by an equal amount of the solution consisting of 40 parts of ethanol, 58 parts of Ringer's solution and 2 parts of formalin. Smears of centrifuged sediments were usually fixed with etheralcohol. The Millipore filter technique was also carried out.

Diagnosis rate for entire intracranial tumors was 41.2 per cent by cerebrospinal fluid. Concerning gliomas, the rate was 37.5 per cent. It was histologically confirmed that the ventricular wall was more or less involved by tumor. By needle aspiration technique the rate was remained 37.5 per cent because of difficulty of selective puncture. According to classic Papanicolaou's classification, class 3 belongs to suspicious group. However the meaning of class 3 must be extended to positive in the central nervous system, because the cases of benign tumor such as astrocytoma below grade 2, pituitary tumor, benign ependymoma etc. were included in class 3 .

On false positive case was that of acoustic neurinoma containing large nucleus with prominent nucleoli.

Millipore filter method was considered to be an excellent method because of high cellular concentration.

\title{
3. Relationships Between the Malignancy of Astrocytomas (Kernohan) and It's Seriographic Findings
}

\author{
Yoshitaka NaKada, Yutaka Maki, Takashi Horie, Satoru AKashI, \\ Shizuo Shirai and Yutaka Matsumoto \\ Neuro-psychiatric Dept., School of Medicine, Chiba Univ.
}

We discussed about the serioangiographic findings on the Grade 1 to 4 astrocytomas, which is due to the classification proposed by J.W. Kernohan. The materials used for discussion were 26 cases of supratentorial astroytomas (Kernohan). We obtained tumor stainings in 18 cases or about $70 \% ; 11$ were of Grade 4 astrocytomas (6), 8 were of Grade 3 (7), one was of Grade 2 (1) and 6 were of Grade 1 (4). The number in the parenthesis means the case in which we obtained tumor stainings.

Tumors, which were localized deep in the midline or about the ventricles, appeared less frequently on angiograms, but those which were located near the cerebral cortex stained well whether or not they were malignant.

We discussed about; 1 ) A-V fistulas, 2) Irregularities concerning the abnormal vessels of the tumors, 3) Hypervascularity, 4) Ratio of tumor stainings in the venous phase. 


\title{
Resulst
}

A-V fistulas were noticed in all case of Grade 4 astrocytomas and most of Grade 3. The nature of the vessles were, except for a few cases, usually irregular in their calivers and pattern. Hypervascularity was prominent in Grade 3, and in Grade 4 it was less than moderate and almost indistinguishable. In Grade 1, hypervascularity was prominent in some cases and was very scarce in others. The ratio of tumor stainings in the venous phase was high in certain cases of Grade 1, in all cases of Grades 2 and 3. The duration of tumor stainings thus decreased in time in the venous phase, so the ratio of the tumor stainings in the venous phase was relatively low in Grade 4.

The angiographical findins of astrocytomas in each Grade are shown on the table below. As we could not get more than one case of Grade 1 astrocytoma, it was omitted in this table.

As shown in the table, Grade 1 and Grade 3 astrocytomas seemed to be able to divide into two subdivisions angiographically. With the usage of seriography the Grades of astrocytomas (Kernohan) could be differentiated to some extent.

\author{
4. Brain Scans in Diagnosis of Brain Tumors \\ (Second Report)
Yutaka MAKI, Yoshitaka NAKadA, Motoyoshi SHIMAZAKI, Yukio Ono, Susuma Minei, Hiroshi Akimoto, Tadabumi KusaKa and Morimasa KoNo \\ Neuropsychiatric Department, Chiba University, School of Medicine \\ Hirotake KAKEHI \\ Department of Radiology, Chiba University, School of Medicine
}

Histologically verified 47 cases with accurate localization were studied on 1) comparison of scans with ${ }^{99 m} \mathrm{TcC}_{4}^{-}$and Chlormerodrin $\mathrm{Hg}-203$, and 2) comparison of brain scannings and serial angiograms.

In 47 cases, scan localized 31 of them $(66 \%), 39$ patients with Chlormerodrin $\mathrm{Hg}-203$ had positive scans of 21 cases $(5 \%)$, but scan with ${ }^{99 m} \mathrm{TcC}_{4}^{-}$ (13 scans) localized 11 cases $(85 \%)$. Serial angiograms were abnormal in 40 cases out of $47(85 \%)$.

Certain tumors such as glioblastoma (11/12) and meningoma (6/6) gave the highest incidence of satisfactory results. 3 cases, glioblastoma, spongioblastoma and metastatic tumor, with normal serial angiograms, revealed positive scans. 